\title{
Phase space evolution of pairs created in strong electric fields
}

\author{
A. Benedetti ${ }^{\mathrm{a}, \mathrm{b}, \mathrm{c}, *}$, R. Ruffini ${ }^{\mathrm{a}, \mathrm{b}, \mathrm{c}}, \mathrm{G}$. V. Vereshchagin ${ }^{\mathrm{a}, \mathrm{b}, * *}$ \\ ${ }^{a}$ ICRANet p.le della Repubblica, 10, 65100 Pescara, Italy \\ ${ }^{b}$ University of Rome "Sapienza", Physics Department, p.le A. Moro 5, 00185 Rome, Italy \\ ${ }^{c}$ ICRANet, Université de Nice Sophia Antipolis, Grand Château, BP 2135, 28, avenue de Valrose, 06103 NICE CEDEX 2, France
}

\begin{abstract}
We study the process of energy conversion from overcritical electric field into electron-positron-photon plasma. We solve numerically Vlasov-Boltzmann equations for pairs and photons assuming the system to be homogeneous and anisotropic. All the 2-particle QED interactions between pairs and photons are described by collision terms. We evidence several epochs of this energy conversion, each of them associated to a specific physical process. Firstly pair creation occurs, secondly back reaction results in plasma oscillations. Thirdly photons are produced by electron-positron annihilation. Finally particle interactions lead to completely equilibrated thermal electron-positron-photon plasma.
\end{abstract}

N

Keywords: Electron-positron plasmas, Kinetic theory

\section{Introduction}

Quantum electrodynamics predicts that vacuum breakdown in a strong electric field $E$ comparable to the critical value $E_{c}=m_{e}^{2} c^{3} / e \hbar$ where $m_{e}$ is the electron mass, $e$ is its charge, $c$ is the speed of light and $\hbar$ is the Planck constant results in non-perturbative electron-positron pair production [1, 2, 3]. Nonlinear effects in high intensity fields can be observed already in undercritical electric field, 'see e.g. [4, 5]. Considerable effort has been made over last two decades in increasing the intensity of high power lasers in order to explore these high field regimes. Yet, the Schwinger field $E_{c}$ is far from being reached, see e.g. for recent review [6]. There are indications that such technology is limited to undercritical fields due to occurrence of avalanches [7, 8] which deplete the external field faster than it can potentially grow. Some authors [9] went that far as to claim that "the critical QED field strength can be never attained for a pair creating electromagnetic field".

While dynamical mechanisms involving increase of initially small electric field toward its critical value appear problematic because of avalanches, existence of overcriti-cal electric field in which pair production is blocked [10] is widely discussed in astrophysical context in compact stars, e.g. hypothetical quark stars [11, 10], neutron stars [12], see also [13] for review. Pair production in such overcritical field may occur due to several reasons, e.g. heating [14] or gravitational collapse of the compact object. Assuming existence of such overcritical electric field in this paper we

\footnotetext{
*Principal corresponding author

${ }^{* *}$ Corresponding author

Email addresses: alberto.benedetti@icra.it (A. Benedetti), ruffini@icra.it (R. Ruffini), veresh@icra.it (G. V. Vereshchagin)
}

revisit the issue of conversion of energy from initial electric field into electron-positron plasma once the blocking is released.

The most general framework for considering the problem of back reaction of created matter fields on initial strong electric field is QED. Up to now the problem has been treated in QED in $1+1$ dimension case for both scalar [15] and fermion [16] fields. It was shown there that pair creation is followed by plasma oscillations due to back reaction of pairs on initial electric field. The results were compared with the solutions of the relativistic Vlasov-Boltzmann equations and shown to agree very well. The Vlasov type kinetic equation for description of $e^{+} e^{-}$plasma creation under the action of a strong electric field was used previously e.g. in the following works [17, 18, 19]. The back reaction problem in this framework was considered by [20, 21]. The kinetic theory to description of the vacuum quark creation under action of a supercritical chromo-electromagnetic field was applied by [22, 23]. Kinetic equations for electron-positron-photon plasma in strong electric field were obtained in [24] from the Bogoliubov-Born-Green-Kirkwood-Yvon hierarchy.

Much simpler model was developed later, starting from Vlasov-Boltzmann equations 25] and assuming that all particles are in the same momentum state at a given time, that allowed to consider pair-photons interactions. In this way the system of partial integro-differential equations was reduced to the system of ordinary differential equations which was integrated numerically. This model was studied in details in [26, 27], where existence of plasma oscillations was confirmed and extended to undercritical electric fields. It was also shown that photons are generated and reach equipartition with pairs on a time scale much longer than the oscillation period. 
In this work for the first time we study the entire dynamics of energy conversion from initial strong electric field, ending up with thermalized electron-positron-photon plasma which is assumed to be optically thick. With this goal we generalize previous treatments [15]-27]. In particular, we relax the delta-function approximation of particle momenta adopted in [25]. In contrast, we obtain the system of partial integro-differential equations which is solved numerically on large timescales, exceeding many orders of magnitude several characteristic timescales of the problem under consideration.

We adopt a kinetic approach in which collisions can be naturally described, assuming invariance under rotations around the direction of the electric field. In this perspective we solve numerically the relativistic VlasovBoltzmann equations with collision integrals computed from the exact QED matrix elements for the two particle interactions 28], namely electron-positron annihilation into two photons and its inverse process, Bhabha, Möller and Compton scatterings.

The paper is organized as follows. In the next section we introduce the general framework, then we report our results. Conclusions follow. Details about the computation are presented in the Appendix.

\section{Framework}

Based on the symmetry of the problem we consider axially symmetric momentum space. Hence, the momentum of the particle is described by two components, one parallel $\left(p_{\|}\right)$and one orthogonal $\left(p_{\perp}\right)$ to the direction of the initial electric field. Then the azimuthal angle $(\phi)$ describes the rotation of $p_{\perp}$ around $p_{\|}$. These momentum space coordinates are defined in the following intervals $p_{\|} \in(-\infty,+\infty), p_{\perp} \in[0,+\infty), \phi \in[0,2 \pi]$. Within the chosen phase space configuration, the prescription for the integral over the entire momentum space is $\int d^{3} \mathbf{p} \quad \rightarrow \quad \int_{0}^{2 \pi} d \phi \int_{-\infty}^{+\infty} d p_{\|} \int_{0}^{+\infty} d p_{\perp} p_{\perp}$ and the relativistic energy is given by the following equation

$$
\epsilon=\sqrt{p_{\|}^{2}+p_{\perp}^{2}+m^{2}}
$$

where $m$ is the mass of the considered particle. In the previous equation and hereafter we set $c=\hbar=1$.

In the adopted kinetic scheme the Distribution Function (DF) is the basic object we are dealing with and all the physical quantities can be extracted from it. Denoting with $\nu$ the kind of particle, the $\operatorname{DF} f_{\nu}$ is commonly used in textbooks such that the corresponding number density is given by its integral over the entire momentum space

$$
n_{\nu}=\int d^{3} \mathbf{p} f_{\nu}=2 \pi \int_{-\infty}^{+\infty} d p_{\|} \int_{0}^{+\infty} d p_{\perp} p_{\perp} f_{\nu} .
$$

Due to the assumed axial symmetry, $f$ does not depend on $\phi$ and consequently it is a function of the two components of the momentum only $f_{\nu}=f_{\nu}\left(p_{\|}, p_{\perp}\right)$. In this paper we use $F_{\nu}=2 \pi \epsilon f_{\nu}$ which allows to write down BoltzmannVlasov equations in conservative form 29, 28], essential for numerical computations. The energy density for each type of particle is given by its integral over the parallel and orthogonal component of the momentum

$$
\rho_{\nu}=\int_{-\infty}^{+\infty} d p_{\|} \int_{0}^{+\infty} d p_{\perp} F_{\nu}
$$

In isotropic momentum space this DF is reduced to the spectral energy density $d \rho_{\nu} / d \epsilon$.

The time evolution of electron and positron DFs is described by the relativistic Boltzmann-Vlasov equation

$$
\begin{aligned}
& \frac{\partial F_{ \pm}\left(p_{\|}, p_{\perp}\right)}{\partial t} \pm \operatorname{eE} \frac{\partial F_{ \pm}\left(p_{\|}, p_{\perp}\right)}{\partial p_{\|}}= \\
& =\sum_{q}\left(\eta_{ \pm}^{* q}\left(p_{\|}, p_{\perp}\right)-\chi_{ \pm}^{q}\left(p_{\|}, p_{\perp}\right) F_{ \pm}\left(p_{\|}, p_{\perp}\right)\right)+ \\
& \quad+S\left(p_{\|}, p_{\perp}, E\right)
\end{aligned}
$$

where $\eta_{ \pm}^{* q}, \chi_{ \pm}^{q}$ are the emission and absorption coefficients due to the interaction denoted by $q$, and the source term $S$ is the rate of pair production. The sum over $q$ covers all the 2-particle QED interactions considered in this work. In particular the electron-positron DFs in Eq. (4), varies due to the acceleration by the electric field, the creation of pairs due to vacuum breakdown and the interactions, see Appendix A.1 for details. Indeed, the Vlasov term describes the mean field produced by all particles, plus the external field. In our approach particle collisions, including Coulomb ones, are taken into account by collision terms. Particle motion between collisions is assumed to be subject to external field only, and the mean field is neglected. This is an assumption, but in dense collision dominated plasma such as the one considered in this paper this assumption is justified, see e.g. [30]. The rate of pair production already distributes particles in the momentum space according to [13]

$$
\begin{aligned}
& S\left(p_{\|}, p_{\perp}, E\right)=-\frac{|e E|}{m_{e}^{3}(2 \pi)^{2}} \epsilon p_{\perp} \times \\
& \times \log \left[1-\exp \left(-\frac{\pi\left(m_{e}^{2}+p_{\perp}^{2}\right)}{|e E|}\right)\right] \delta\left(p_{\|}\right) .
\end{aligned}
$$

For $E<E_{c}$ this rate is exponentially suppressed. Besides, Eq. (5) already indicates that pairs are produced with orthogonal momentum, up to about $m_{e}\left(E / E_{c}\right)$ but at rest along the direction of the electric field.

The Boltzmann equation for photons is

$$
\frac{\partial F_{\gamma}\left(p_{\|}, p_{\perp}\right)}{\partial t}=\sum_{q}\left(\eta_{\gamma}^{* q}\left(p_{\|}, p_{\perp}\right)-\chi_{\gamma}^{q}\left(p_{\|}, p_{\perp}\right) F_{\gamma}\left(p_{\|}, p_{\perp}\right)\right)
$$

and their DF changes due to the collisions only. In more detail, photons must be produced first by annihilating pairs, then they affect the electron-positron DF through Compton scattering. Besides, also photons annihilation 
into electron-positron pairs becomes significant at later times. Eqs. (6) and (44) are coupled by means of the collision integrals, therefore they are a system of partial integro-differential equations that must be solved numerically. Efficient method for solving such equations in optically thick case was developed in [29] and later generalized in [28], see Appendix A.2 and Appendix A.3 for details.

It is well known [25, 26] that both acceleration and pair creation terms in Eq. (4) operate on a much shorter timescale than interactions with photons described by collision terms in Eqs. (44) and (6). For this reasons we run two different classes of simulations, one neglecting collision integrals which is referred to as "collisionless" and another one including them called "interacting".

\section{Results}

In this section we describe our results for the collisionless and interacting systems separately. The boundary condition is set when the initial electric field $E_{0}$ and the initial DF $F_{\nu 0}\left(p_{\|}, p_{\perp}\right)$ are specified. For simplicity we performed several runs with different initial electric fields, but always with no particles at the beginning. When in addition to external electric field also particles are present from the beginning, oscillations still occur, but with higher frequency, as given by the plasma frequency [27]. So we start computations with DFs null identically in the whole momentum space and our initial conditions can be written as

$$
\left\{\begin{array}{l}
E_{0}=\xi E_{c}, \quad \xi=\{1,3,10,30,100\} \\
F_{\nu 0}\left(p_{\|}, p_{\perp}\right)=0, \quad p_{\perp} \in[0,+\infty), p_{\|} \in(-\infty,+\infty) .
\end{array}\right.
$$

Consequently electrons and positrons are produced exclusively by the Schwinger process.

In order to interpret meaningfully our results, we introduce first some useful quantities. Initially the energy is stored in the electric field and it fixes the energy budget available as given by

$$
\rho_{0}=\frac{E_{0}^{2}}{8 \pi} .
$$

We expect therefore the final state of the equilibrated thermal electron-positron-photon plasma to be characterized by the temperature

$$
T_{e q}=\sqrt[4]{\frac{\rho_{0}}{4 \sigma}} \simeq 1.7 \sqrt{\frac{E_{0}}{E_{c}}} \mathrm{MeV}
$$

where $\sigma$ is the Stefan-Boltzmann constant. The total energy density of pairs $\rho_{ \pm}$and photons $\rho_{\gamma}$ are related to the actual and initial electric fields, $E$ and $E_{0}$, by the energy conservation law

$$
\rho_{ \pm}=\rho_{+}+\rho_{-}=\frac{E_{0}^{2}-E^{2}}{8 \pi}-\rho_{\gamma}
$$

Following [27], we define the maximum achievable pairs number density

$$
n_{\max }=\frac{E_{0}^{2}}{8 \pi m_{e}}
$$

which corresponds to the case of conversion of the whole initial energy density into electron-positron rest energy density

$$
\rho_{ \pm r e s t}=\left(n_{-}+n_{+}\right) m_{e}
$$

where $n_{-}$and $n_{+}$are the electrons and positrons number densities respectively. From the electrons and positrons DFs we can extrapolate their bulk parallel momentum $\left\langle p_{\|}\right\rangle$ as defined in Eq. A.5 and the symmetry of our problem implies that $\left\langle p_{\|-}\right\rangle=-\left\langle p_{\|+}\right\rangle$. We make use of this identity to define the kinetic energy density of pairs

$$
\rho_{ \pm k i n}=\rho_{ \pm r e s t}\left(\sqrt{\left(\frac{\left\langle p_{\|_{ \pm}}\right\rangle}{m_{e}}\right)^{2}+1}-1\right)
$$

Therefore $\rho_{ \pm k i n}$ is the energy density as if all particles are put together in the momentum state with $p_{\|}=\left\langle p_{\|}\right\rangle$and $p_{\perp}=0$ while their rest energy density is $\rho_{ \pm r e s t}$. The difference between the total energy density and all the others defined above is denoted as internal energy density

$$
\rho_{ \pm i n}=\rho_{ \pm}-\rho_{ \pm r e s t}-\rho_{ \pm k i n} .
$$

The term "internal" refers here to the dispersion of the DF around a given point with coordinates $\left(\left\langle p_{\|}\right\rangle,\left\langle p_{\perp}\right\rangle\right)$ in the momentum space.

\subsection{Collisionless systems}

Since interactions with photons operate on much larger time scale than the pair creation by vacuum breakdown we first present the results obtained solving the relativistic Boltzmann equation (4) for electrons and positrons with $\chi_{ \pm}^{q}=\eta_{ \pm}^{* q}=0$. With these assumptions we expect the following results to be closely related to those reported in [27].

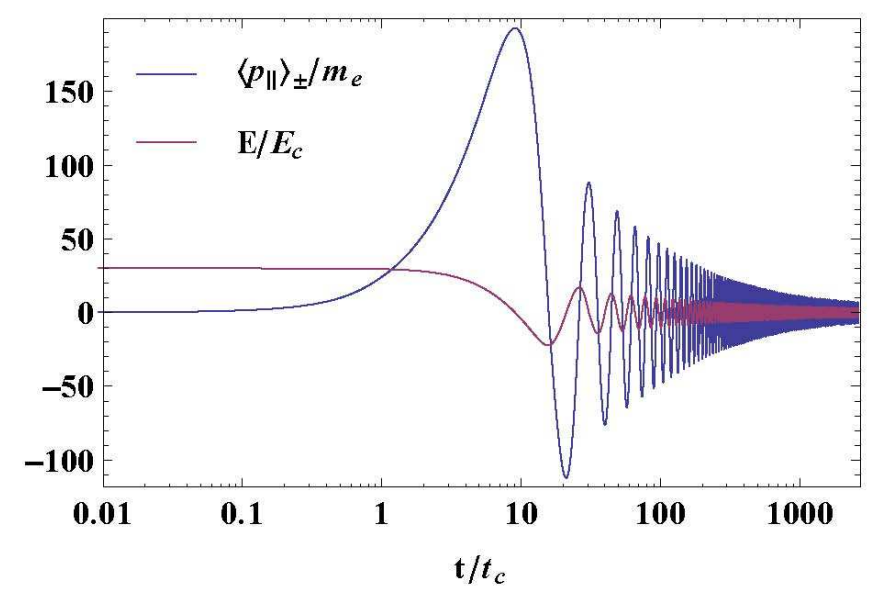

Figure 1: Evolution of electric field $E$ and pairs bulk parallel momentum $\left\langle p_{\|}\right\rangle_{ \pm}$obtained from the numerical solution of Eq. (4) setting $E_{0}=30 E_{c}$. 


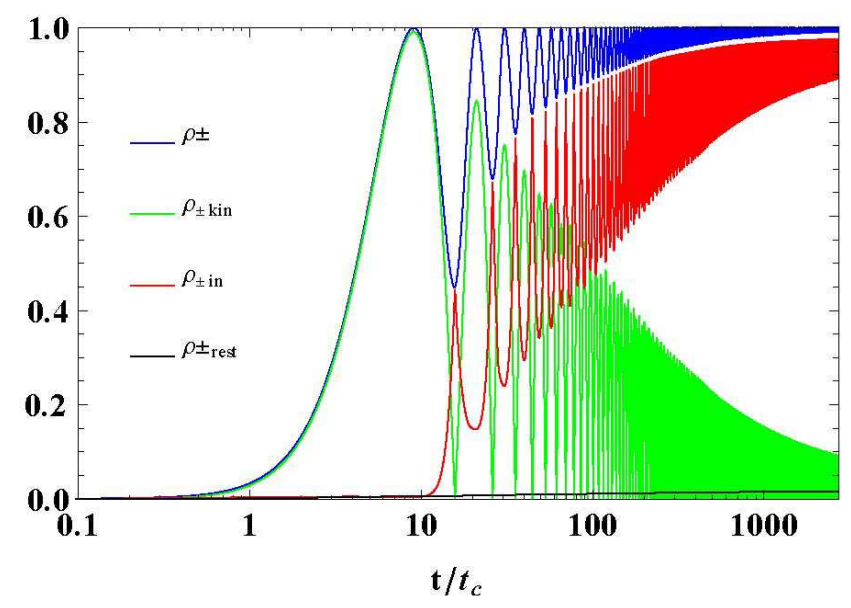

Figure 2: Evolution with time of the pairs energies as defined by Eqs. (9), (11), (12) and (13) for the collisionless case $E_{0}=30 E_{c}$. All of them are normalized by the total initial energy $\rho_{0}$ given by Eq. (7).

For all the explored initial conditions, there are important analogies between the approach adopted in [27] and the one presented in this work. For each initial field the first half period of the oscillation $t_{1}$ is nearly equal to the corresponding one obtained in [27]. Also the evolution with time of $\left\langle p_{\|}\right\rangle_{ \pm}$during this time lapse is very similar to the result given by their analytic method. The time evolution of electric field $E$ and $\left\langle p_{\|}\right\rangle_{ \pm}$in Compton units with $t_{c}=1 / m_{e}$ are shown in Fig. 1] for $E_{0}=30 E_{c}$.

In addition to these similarities some new important features emerge from the current study. The manifestation of these new aspects is represented in Fig. 2 where we show how the various forms of $e^{ \pm}$energy defined in the previous paragraph evolve with time. These energy densities are normalized to the total initial energy density $\rho_{0}$ defined by Eq. (7). One of the most important evidences of this figure is that the rest energy density of pairs $\rho_{ \pm \text {kin }}$ saturates to a small fraction of the maximum achievable one. This is in contrast with the result presented in [27] where the value given in Eq. (10) was reached asymptotically.

As a consequence the energy is mainly converted into other forms, namely the kinetic $\rho_{ \pm \text {kin }}$ and internal $\rho_{ \pm i n t}$ ones. Both these quantities oscillate with the same frequency but with shifted phase. Relative maxima and minima of $\rho_{ \pm k i n}$ correspond to the peaks of the bulk parallel momentum shown in Fig. 1 as can be grasped from its definition in Eq. (12). Looking at Fig. 2 we see their relative importance changing progressively with time. Even if they oscillate, the internal component dominates over the kinetic one as time advances. This trend points out that all the initial energy will be converted mostly into internal energy, while the contribution of the kinetic one will eventually be small.

In this respect, from the electron and positron DFs we obtain the mean squared values of the parallel and orthogonal momentum as defined by Eqs. (A.6) and (A.7). These
Table 1: Square root of the mean squared value of orthogonal $\left\langle p_{\perp}^{2}\right\rangle_{ \pm}$ and parallel $\left\langle p_{\|}^{2}\right\rangle_{ \pm}$momentum, parallel momentum $p_{\| 1}$, in units of $m_{e}$, and number density $n_{1}$ of pairs at the first zero of the electric field, saturation number density $n_{s}$ normalized by the maximum achievable one given by Eq. 10 for different initial electric fields.

\begin{tabular}{|c|c|c|c|c|c|}
\hline$\frac{E}{E_{c}}$ & $\sqrt{\left\langle p_{\perp}^{2}\right\rangle_{ \pm}}$ & $\sqrt{\left\langle p_{\|}^{2}\right\rangle_{ \pm}}$ & $\left\langle p_{\|}\right\rangle_{1}$ & $\frac{n_{1}}{n_{\max }}$ & $\frac{n_{s}}{n_{\max }}$ \\
\hline \hline 1 & 0.4 & 75 & 160 & 0.006 & 0.018 \\
\hline 3 & 0.8 & 37 & 82 & 0.018 & 0.037 \\
\hline 10 & 1.3 & 35 & 77 & 0.013 & 0.041 \\
\hline 30 & 2.0 & 87 & 192 & 0.005 & 0.016 \\
\hline 100 & 3.5 & 127 & 284 & 0.003 & 0.011 \\
\hline
\end{tabular}

quantities give us some insight about the spreading of the DF along the parallel and orthogonal components of the momentum. In Tab. 1 we report their values at the end of runs with different initial fields. It is clear that the larger the initial electric field the larger is $\left\langle p_{\perp}^{2}\right\rangle_{ \pm}$. This is a direct consequence of the rate of pair production given by Eq. (5) that already distributes particle along the orthogonal direction in the momentum space.

The mean squared value of the parallel momentum $\left\langle p_{\|}^{2}\right\rangle_{ \pm}$reaches a minimum value between 3 and 10 critical electric fields. This minimum was first found in [26], see Fig. 3 in that paper. In Tab. 1 we report also $\left\langle p_{\|}\right\rangle_{ \pm 1}$ which is the peak value of the bulk parallel momentum at the moment when electric field vanishes for the first time. We see from the table that also this quantity has a minimum in the same range of initial fields as $\left\langle p_{\|}^{2}\right\rangle_{ \pm}$. Both these minima are linked to the combined effects of pairs creation and acceleration processes.

However, it is important to compare $\left\langle p_{\|}^{2}\right\rangle_{ \pm}$and $\left\langle p_{\perp}^{2}\right\rangle_{ \pm}$for different initial fields. Indeed, this juxtaposition gives us quantitative informations about the anisotropy of the DFs in the phase space. Looking at the numerical values we observe how this anisotropy decreases with the increase of the initial electric field, which points out how an eventual approach toward isotropy, and therefore thermalization, would be much more difficult for lower initial fields.

In Tab. 1 we compare also two different number densities $n_{1}$ and $n_{s}$ normalized to the maximum achievable one. The first is the number density of pairs at the first zero of the electric field $t_{1}$. The second is the saturation number density of pairs at the end of the run. We found that the values of $n_{1}$ are very close to the same densities computed in [27] with a significant amount of pairs produced already in a very small time lapse. Let us note that there are maxima of both $n_{1}$ and $n_{s}$ in the range between 1 and $10 E_{c}$ in correspondence with minima of $\left\langle p_{\|}^{2}\right\rangle_{ \pm}$and $\left\langle p_{\perp}^{2}\right\rangle_{ \pm}$ 


\subsection{Interacting systems}

Now we turn to the dynamics of our system on much larger time scales. As discussed above, in long run interactions between created pairs become important. We consider 2-particle interactions listed in Tab. A.2 in Appendix and describe them by the collision integrals in Eqs. (4) and (6) using the same range of initial fields used for the collisionless systems. More sparse computational grid is used as calculation of collision terms imply performing multidimensional integrals in the phase space, see Appendix Appendix A.2.

The larger the electric field the higher the rate of pairs production and consequently their number density. Since the interaction rate is proportional to particle number densities, we expect them to be important sooner for higher initial field. In this respect, it is worth mentioning that in [27] the time $t_{\gamma}$ was estimated at which the optical depth for electron-positron annihilation equals unity $\tau\left(t_{\gamma}\right)=1$. There, it was found that $t_{\gamma}$ decreases when the initial electric field increases. Besides, the order of magnitude of their estimations is in agreement with the time at which the photons number density is around a few percent of the pairs number density.

In the previous subsection dedicated to collisionless systems, we described the anisotropy of the pairs DF by means of the mean squared value of parallel and orthogonal momentum reported in Tab. 1]. In that case, we knew approximately the range of orthogonal momentum in which the most part of electrons and positron were located and the orthogonal grid was chosen and kept fixed from the beginning. This choice was possible because the dispersion along the orthogonal direction was determined uniquely by the rate $S$. The extension of the grid was chosen in such a way that the value of each DF at the grid boundaries was small compared to the maximum value. For the reason that interactions redistribute particles in the phase space and tend to isotropize their distributions, the orthogonal grid must be extended to values comparable to the kinetic equilibrium temperature. To do that, we use initially an orthogonal grid with the same extension as in the collisionless system. We extend it later when particles are scattered toward higher orthogonal momenta and therefore the tails of the DF at the boundaries is not negligible. The extension of the parallel grid remains essentially the same as the collisionless case.

In order to correctly describe the pairs acceleration process, the time step of the computation must be a small fraction of their oscillation period. This constraint prevents us to study the evolution up to the kinetic equilibrium within a reasonable time. After hundreds of oscillations, the energy density carried by the electric field is a small fraction of the pairs and photons energy densities. In other words, most energy has already been converted into electron-positron plasma. Due to this fact, the acceleration of electrons and positrons does not affect their DFs appreciably. This allows us to neglect the presence

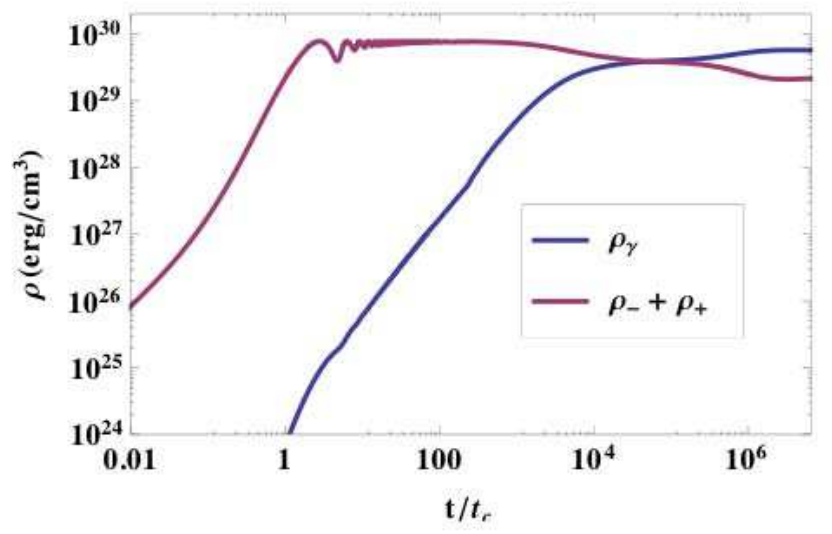

Figure 3: Energy densities of pairs and photons obtained from the numerical solution of Eqs. (4) and (6) with initial field $E_{0}=100 E_{c}$.

of the electric field hereafter. To do that we use the distribution function at this instant as initial condition for a new computation in which the condition $E=0$ is imposed. By neglecting oscillations induced by the electric field the constraint on the time step of the numerical calculation is released, and it is now determined by the rate of the interactions.

In Fig. 3 we show the time evolution of the pairs and photons energy densities for $E_{0}=100 E_{c}$. From this plot we can understand the hierarchy of time scales associated to the distinct physical phenomena we are dealing with. In presence of an overcritical electric field, electron-positron pairs start to be produced in a shortest time according to Eq. (5). As soon as they are created, electrons and positrons are accelerated toward opposite directions as the back reaction effect on the external field. The characteristic duration of this back reaction corresponds approximately to the first half oscillation period. At early times, even after many oscillations, the energy density of photons is negligible compared to that of pairs, meaning that interactions do not play any role. Such a starting period, during which the real system can be considered truly collisionless, exists independently on the initial electric field even if its duration depends on it. From Fig. 3 it is clear that the photons energy density increases with time as a power law approaching the pairs energy density.

Only when hundreds oscillations have taken place, interactions start to affect the evolution of the system appreciably and can not be neglected any further. The slope of the photons curve in 3 reduces indicating that pairs annihilation has become less efficient than the photons annihilation process. Now the evolution of the system is mostly governed by interactions. Möller, Bhabha and Compton scatterings give rise to momentum and energy exchange between electron, positron and photon populations. Besides the same collisions have the tendency to distribute particles more isotropically in the momentum space. After some time, the photons energy density becomes equal 

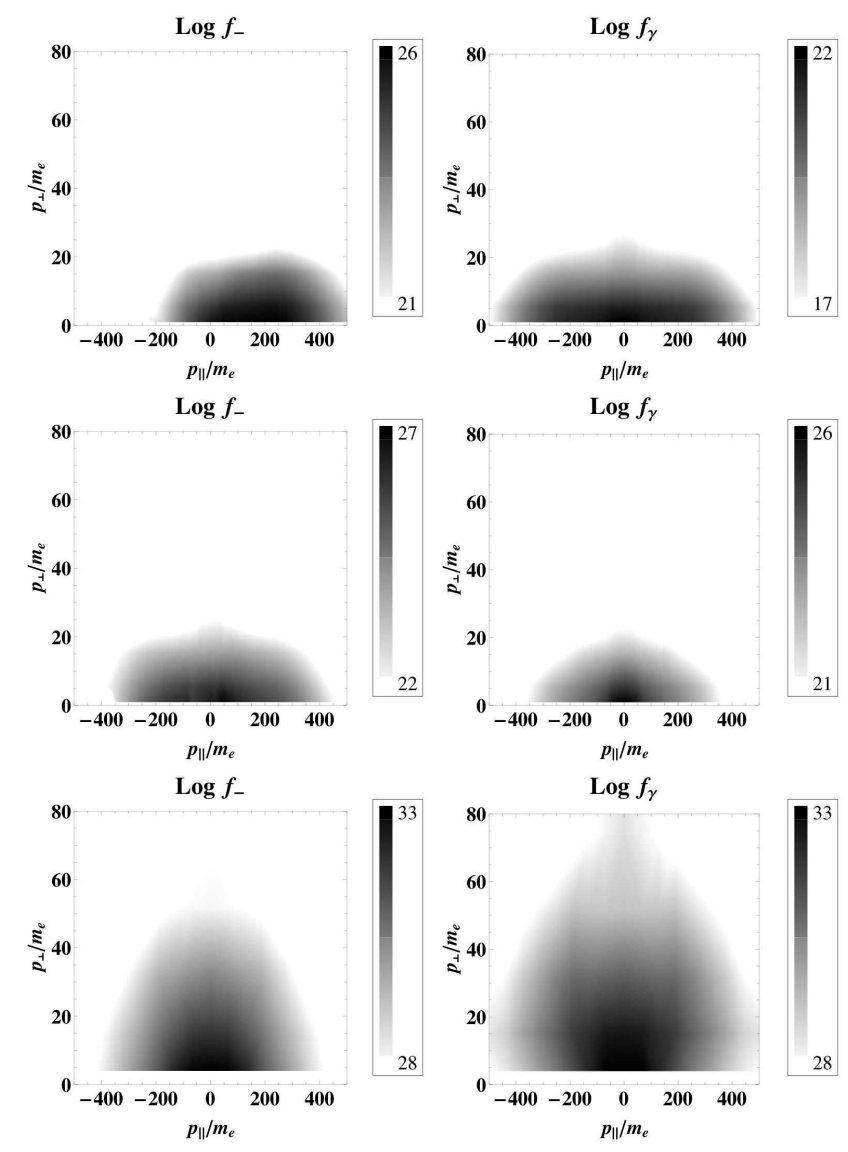

Figure 4: Phase space distributions of electrons (left column) and photons (right column) for the initial condition $E_{0}=100 E_{c}$. Top: $2.3 \cdot 10^{2} t_{c}$, middle: $2.3 \cdot 10^{2} t_{c}$, bottom: $4.6 \cdot 10^{6} t_{c}$.

and then overcomes the pairs energy density. This growth continues until the equilibrium between pairs annihilation and creation processes is established $e^{-} e^{+} \leftrightarrow \gamma \gamma$. For this reason both pairs and photons curve are flat on the right of Fig. 3, see also [31]. However, at this point the DF is not yet isotropic in the momentum space indicating that the kinetic equilibrium condition is not yet satisfied. In fact, kinetic equilibrium is achieved only at later times when also Möller, Bhabha and Compton scatterings are in detailed balance. At that time the electron-positron-photon plasma can be identified by a common temperature and nonzero chemical potential. This is also the last evolution stage attainable by our study because only 2-particle interactions are taken into account while thermalization is expected to occur soon after kinetic equilibrium if also 3 -particle interactions would be included [28].

As an example, in Fig. 4 density plots of $f_{-}$and $f_{\gamma}$ are shown on the left and right columns respectively, for the initial condition $E_{0}=100$. Their time evolution starts from the top line to the bottom one corresponding to three different times. After $2.3 t_{c}$ both DFs are highly anisotropic as it is well established by the ratio $R=\sqrt{\left\langle p_{\|}^{2}\right\rangle_{ \pm} /\left\langle p_{\|}^{2}\right\rangle_{ \pm}}=0.06$. At this stage, the electric field is highly overcritical and a very small fraction of ini- tial energy has been converted into rest mass energy of electrons and positrons. For this reason $e^{-} e^{+}$are easily accelerated up to relativistic velocities explaining why the electron DF is shifted on the right side of the phase space plane characterized by $p_{\|}>0$. At this instant electrons are characterized by a relativistic bulk velocity corresponding to a Lorentz gamma factor 170 . On the second line the time is $2.3 \cdot 10^{2} t_{c}$ and the DFs are still anisotropic if we look at the parameter $\mathrm{R}$ introduced above. However the situation is different with respect to the previous stage because the electric field is only slightly overcritical and many more pairs and photons have been generated. As a consequence interaction rates are much larger than before and an efficient momentum exchange between electron and positron populations occurs. Both small electric field and collisions prevent particles to reach ultra-relativistic velocities and for this reason the electron DF is now perfectly symmetric with respect to the plane $p_{\|}=0$. Only later on, at $4.6 \cdot 10^{6} t_{c}$ for the bottom line, collisions dominate the evolution of the system whereas the presence of the electric field can be safely neglected. The pictures show a prominent DFs widening toward higher orthogonal momenta which is confirmed by the value $R \simeq 0.23$. This remarkable evidence allows us to predict the forthcoming fate of the system to be an electron-positron-photon plasma in thermal equilibrium. The DFs isotropy in the momentum space not only indicates that the kinetic equilibrium condition is approached but also that the system is going to lose information about the initial preferential direction of the electric field. In the case of isotropic DF, the timescale on which thermal equilibrium is achieved can be estimated as $\tau_{t h} \simeq 1 /\left(n \sigma_{T}\right)$ [28]. For our anisotropic $\mathrm{DF}$ the thermalization timescale is remarkably longer.

\section{Conclusions}

From the conceptual point of view the discovery of plasma oscillations [15] as result of back reaction has been an important step. The next step has been the analysis of creation of photons from pairs 25]. In this paper we have studied all these phenomena in great details adopting a kinetic approach with two dimensional phase space. We have found anisotropy in momentum distributions of pairs and photons, which we consider one of the main results of this paper. Another important result is the importance of internal energy which limits heavily the efficiency of energy conversion into electron-positron pairs. Both these effects could be in principle considered as phenomenological evidences of an overcritical electric field, even if they manifest themselves on a very short time scale.

For the first time we studied the entire dynamics of energy conversion from initial overcritical electric field, ending up with thermalized electron-positron-photon plasma. Such conversion occurs in a complicated sequence of processes starting with Schwinger pair production which is followed by oscillations of created pairs due to back-reaction on initial electric field, then production of photons due 
to annihilation of pairs and finally isotropization of created electron-positron-photon plasma. We solved numerically the relativistic Vlasov-Boltzmann equations for electrons, positrons and photons, with collision integrals for 2-particle interactions computed from exact QED matrix elements.

In order to appreciate the consequences of the kinetic treatment and the relevance of interactions separately, two different computations have been performed for every single initial condition. We called them collisionless and interacting systems in view of the fact that collision terms have been discarded and accounted for, respectively. The collisionless runs allowed us to compare our results with those obtained earlier, and to resolve better the momentum space of pairs. In this way we found that number density of pairs always saturates without exceeding 5 per cent of the maximum achievable number density (10), in contrast to earlier works. This number is not far from the thermal number density of pairs obtained from the temperature (8). In particular, for the largest field $E_{0}=100 E_{c}$ we obtained almost 30 per cent of the thermal number density of pairs when the interactions are not yet important. It is interesting that the energy stored in initial electric field is mainly converted into internal and kinetic energies of pairs, but the former becomes predominant as time advances. Even if the distribution in momentum space reminds Maxwellian, also at the very beginning it is highly anisotropic, with the dispersion along the direction of electric field exceeding orders of magnitude that in orthogonal direction. We conclude that simultaneous production of pairs and their acceleration in the same electric field is responsible for such peculiar form of DF of particles.

We found that interactions become important at later times with respect to the average oscillation period, in agreement with estimates performed in [27]. For higher initial fields interactions become significant earlier and for each initial condition there is a characteristic time scale after which they can not be neglected. We find that photons initially follow the distribution of pairs with nonzero parallel bulk momentum.

The first equilibrium manifests itself when the perfect symmetry between pair annihilation and creation rates is established. Only later on, when scatterings have distributed particles isotropically in the momentum space, the kinetic equilibrium is reached. In such state the electron-positron-photon plasma is generally described by a common temperature and nonzero chemical potentials for all particles and its evolution toward thermal equilibrium is well understood [31].

\section{Appendix A. Computational scheme}

The discretization of the phase space is done defining a finite number of elementary volumes which are uniquely identified by triplets of integer numbers $(i, k, l)$. Their values run over the ranges $\{1,2, \ldots, I-1, I\},\{1,2, \ldots, K-1, K\}$ and $\{1,2, \ldots, L-1, L\}$ respectively. Since we are dealing with an axially symmetric phase space with respect to the direction of the electric field, the parallel momentum is aligned with it while the orthogonal component lays on the plane orthogonal to this preferential axis. Each elementary volume encloses only one momentum vector which can be written explicitly in cylindrical coordinates as $\left(p_{\| i}, p_{\perp k}, \phi_{l}\right)$. The corresponding boundaries are marked by semi-integer indices $\left[p_{\| i-1 / 2}, p_{\| i+1 / 2}\right]$, $\left[p_{\perp k-1 / 2}, p_{\perp k+1 / 2}\right],\left[\phi_{l-1 / 2}, \phi_{l+1 / 2}\right]$. Due to axial symmetry, the DFs do not depend on the azimuthal angle $\phi$ and the index $l$ will be used only to identify angles explicitly. We use also the symbol $\nu$ which identifies the kind of particle under consideration, $\{\gamma,-,+\}$ for photons, electrons and positrons respectively. From these definitions, the energy of a particle with mass $m_{\nu}$ corresponding to the grid point $(i, k)$ is

$$
\epsilon_{\nu i k}=\sqrt{m_{\nu}^{2}+p_{\| i}^{2}+p_{\perp k}^{2}}, \quad\left(m_{\gamma}=0, m_{ \pm}=m_{e}\right)
$$

In this finite difference representation the distribution function has a Klimontovich form and can be seen as a sum of Dirac deltas centered on the grid points $(i, k)$ and multiplied by the energy density of particles on the same grid point $F_{\nu i k}$

$$
F_{\nu}\left(p_{\|}, p_{\perp}\right)=\sum_{i k} \delta\left(p_{\|}-p_{\| i}\right) \delta\left(p_{\perp}-p_{\perp k}\right) F_{\nu i k}
$$

where $\sum_{i k}=\sum_{i=1}^{I} \sum_{k=1}^{K}$. From the definition above and from Eq. (3) the energy and number densities of particle $\nu$ are given by

$$
\begin{aligned}
& \rho_{\nu}=\sum_{i k} F_{\nu i k}, \\
& n_{\nu}=\sum_{i k} n_{\nu i k},
\end{aligned}
$$

where $n_{\nu i k}=F_{\nu i k} / \epsilon_{\nu i k}$. Then the mean parallel momentum, its mean squared value and the mean squared value of the orthogonal momentum are

$$
\begin{aligned}
\left\langle p_{\|}\right\rangle_{\nu} & =\frac{1}{n_{\nu}} \sum_{i k} n_{\nu i k} p_{\| i} \\
\left\langle p_{\|}^{2}\right\rangle_{\nu} & =\frac{1}{n_{\nu}} \sum_{i k} n_{\nu i k}\left(p_{\| i}-\left\langle p_{\|}\right\rangle_{\nu}\right)^{2} \\
\left\langle p_{\perp}^{2}\right\rangle_{\nu} & =\frac{1}{n_{\nu}} \sum_{i k} n_{\nu i k} p_{\perp k}^{2} .
\end{aligned}
$$

Due to axial symmetry the mean orthogonal momentum must be null identically $\left\langle p_{\perp}\right\rangle_{\nu}=0$.

\section{Appendix A.1. Acceleration and electric field evolution}

Once electrons and positrons are produced, they are accelerated by the electric field toward opposite directions. 
The time derivative of the electron or positron parallel momentum $d p_{\| \pm}$in the presence of an electric field $E$ is given by the equation of motion

$$
\frac{d p_{\| \pm}}{d t}= \pm e E
$$

where the sign $+(-)$ refer to the positron (electron) and $-e$ is the electron charge.

Numerically, we move particles from one cell to another one such that the number of particles is conserved and Eq. (A.8) is satisfied. Acceleration causes the changing with time of $F_{ \pm i k}$ which can written as follows

$$
\frac{\partial F_{ \pm i k}}{\partial p_{\|}}= \pm\left(\alpha_{i-1 k} F_{ \pm i-1 k}+\alpha_{i k} F_{ \pm i k}+\alpha_{i+1 k} F_{ \pm i+1 k}\right)
$$

where the coefficients $\alpha_{-, 0,+}$ are defined below

$$
\begin{aligned}
\alpha_{i-1 k} & =\frac{\epsilon_{ \pm i k}}{\epsilon_{ \pm i-1 k}} \frac{1}{p_{\| i}-p_{\| i-1}}, \\
\alpha_{i k} & =\frac{1}{p_{\| i}-p_{\| i-1}}-\frac{1}{p_{\| i+1}-p_{\| i}}, \\
\alpha_{i+1 k} & =\frac{\epsilon_{ \pm i k}}{\epsilon_{ \pm i+1 k}} \frac{1}{p_{\| i+1}-p_{\| i}} .
\end{aligned}
$$

Also the electric field evolves according to the Maxwell equations. Once the currents of the moving pairs are computed, the time derivative of the electric field is known. Consequently a new ordinary differential equation must be added to the system of Eqs. (4) and (6). However, due to the uniformity and homogeneity of the physical space, we can describe the electric field simply using the energy conservation law.

\section{Appendix A.2. Emission and absorption coefficients}

In kinetic theory the time derivative of the $\mathrm{DF} f_{\nu}$ due to interactions between particles is generally written as 32]

$$
\left.\frac{\partial f_{\nu}(\mathbf{p})}{\partial t}\right|_{\text {coll }}=\sum_{q}\left(\eta_{\nu}^{q}(\mathbf{p})-\chi_{\nu}^{q}(\mathbf{p}) f_{\nu}(\mathbf{p})\right)
$$

where $q$ is the label of a specific 2-particle interaction. Eq. A.13 represents a coupled system of partial integrodifferential equations and can be rewritten as follows

$$
\left.\frac{\partial F_{\nu}(\mathbf{p})}{\partial t}\right|_{\text {coll }}=\sum_{q}\left(\eta_{\nu}^{* q}(\mathbf{p})-\chi_{\nu}^{q}(\mathbf{p}) F_{\nu}(\mathbf{p})\right),
$$

where $\eta_{\nu}^{* q}=2 \pi \epsilon p_{\perp} \eta_{\nu}^{q}$. The right hand side of the previous equation contains the so called "collision integrals" used in Eqs. (44) and (6).

In order to describe how the collision integrals are computed, we write down schematically a general 2-particles interaction as which means that particles 1 and 2 having respectively momenta $\mathbf{p}_{1}$ and $\mathbf{p}_{2}$ are absorbed; while in the same process particles 3 and 4 with momenta $\mathbf{p}_{3}$ and $\mathbf{p}_{4}$ are produced. The considered interactions are shown in Table (A.2). From the kinetic theory, the absorption and emis-

Table A.2: Exemplification of the schematic interaction for each of the considered QED 2-particles interactions.

\begin{tabular}{|c|c|c|c|c|}
\hline Interaction & $\mathbf{1}$ & $\mathbf{2}$ & $\mathbf{3}$ & $\mathbf{4}$ \\
\hline \hline Pair Annihilation & $e^{-}$ & $e^{+}$ & $\gamma$ & $\gamma$ \\
\hline Pair Creation & $\gamma$ & $\gamma$ & $e^{-}$ & $e^{+}$ \\
\hline Compton Scattering & $e^{ \pm}$ & $\gamma$ & $e^{ \pm}$ & $\gamma$ \\
\hline Bhabha Scattering & $e^{ \pm}$ & $e^{\mp}$ & $e^{ \pm}$ & $e^{\mp}$ \\
\hline Möller Scattering & $e^{ \pm}$ & $e^{ \pm}$ & $e^{ \pm}$ & $e^{ \pm}$ \\
\hline
\end{tabular}

sion coefficients for the specified process are given by

$$
\begin{aligned}
& \chi_{1}\left(\mathbf{p}_{1}\right) f_{1}\left(\mathbf{p}_{1}\right)= \int d^{3} \mathbf{p}_{2} \int d^{3} \mathbf{p}_{3} \int d^{3} \mathbf{p}_{4} \times \\
& \times w_{1,2 ; 3,4} f_{1}\left(\mathbf{p}_{1}\right) f_{2}\left(\mathbf{p}_{2}\right), \\
& \chi_{2}\left(\mathbf{p}_{2}\right) f_{2}\left(\mathbf{p}_{2}\right)= \int d^{3} \mathbf{p}_{1} \int d^{3} \mathbf{p}_{3} \int d^{3} \mathbf{p}_{4} \times \\
& \times w_{1,2 ; 3,4} f_{1}\left(\mathbf{p}_{1}\right) f_{2}\left(\mathbf{p}_{2}\right), \\
& \eta_{3}\left(\mathbf{p}_{3}\right)=\int d^{3} \mathbf{p}_{1} \int d^{3} \mathbf{p}_{2} \int d^{3} \mathbf{p}_{4} \times \\
& \quad \times w_{1,2 ; 3,4} f_{1}\left(\mathbf{p}_{1}\right) f_{2}\left(\mathbf{p}_{2}\right), \\
& \eta_{4}\left(\mathbf{p}_{4}\right)=\int d^{3} \mathbf{p}_{1} \int d^{3} \mathbf{p}_{2} \int d^{3} \mathbf{p}_{3} \times \\
& \times w_{1,2 ; 3,4} f_{1}\left(\mathbf{p}_{1}\right) f_{2}\left(\mathbf{p}_{2}\right),
\end{aligned}
$$

where the integrals must be calculated all over the phase space. The "transition rate" $w_{1,2 ; 3,4}$ is given by 33]

$$
\begin{gathered}
w_{1,2 ; 3,4}=\frac{1}{(2 \pi)^{2}} \frac{\left|M_{f i}\right|^{2}}{16 \epsilon_{1} \epsilon_{2} \epsilon_{3} \epsilon_{4}} \times \\
\times \delta\left(\epsilon_{1}+\epsilon_{2}-\epsilon_{3}-\epsilon_{4}\right) \delta^{(3)}\left(\mathbf{p}_{1}+\mathbf{p}_{2}-\mathbf{p}_{3}-\mathbf{p}_{4}\right)
\end{gathered}
$$

and it contains all the informations about the probability that such a process occurs. The Dirac Delta's are needed to satisfy the energy and momentum conservation laws.

Eqs. A.15)-(A.18) can be discretized using the integral prescriptions given below

$$
\begin{aligned}
\chi_{1 i_{1} k_{1}} F_{1 i_{1} k_{1}} & =\int_{V_{i_{1} k_{1}}} d^{3} \mathbf{p} \epsilon_{\mathbf{p}} \chi_{1}(\mathbf{p}) f_{1}(\mathbf{p}), \\
\chi_{2 i_{2} k_{2}} F_{2 i_{2} k_{2}} & =\int_{V_{i_{2} k_{2}}} d^{3} \mathbf{p} \epsilon_{\mathbf{p}} \chi_{2}(\mathbf{p}) f_{2}(\mathbf{p}), \\
\eta_{3 i_{3} k_{3}}^{*} & =\int_{V_{i_{3} k_{3}}} d^{3} \mathbf{p} \epsilon_{\mathbf{p}} \eta_{3}(\mathbf{p}), \\
\eta_{4 i_{4} k_{4}}^{*} & =\int_{V_{i_{4} k_{4}}} d^{3} \mathbf{p} \epsilon_{\mathbf{p}} \eta_{4}(\mathbf{p}),
\end{aligned}
$$


where $V_{i_{n} k_{n}}$ is the volume in the phase space which contains only the grid point with $p_{\| i_{n}}$ and $p_{\perp k_{n}}$. It is actually a ring with inner radius $p_{\perp k-1 / 2}$, outer radius $p_{\perp k+1 / 2}$ and thickness $p_{\| i+1 / 2}-p_{\| i-1 / 2}$. An explicit expression for the collision integrals can be obtained inserting Eqs. (A.15)-A.18) into the corresponding Eqs. A.20- A.23). Then we replace all the integrals over the entire momentum space with a sum of integrals over the elementary volumes $V_{i k}$

$$
\int d^{3} \mathbf{p} \rightarrow \sum_{i k} \int_{V_{i k}} d^{3} \mathbf{p}
$$

Even if the sums are different, as well as the integrands, we have exactly the same sequence of integrals in all the emission and absorption coefficients. Due to this fact, we can now adopt the same treatment in order to simplify their expressions.

Let us note first that the interaction cross section is invariant by rotations around an arbitrary axis, therefore one angle can be fixed. In this respect we set $\phi_{1}=0$ and the corresponding integral gives a constant factor $2 \pi$. Dirac Deltas in the transition rate $w_{1,2 ; 3,4}$ are used to eliminate three integrals over $p_{\| 4}, p_{\perp 4}, \phi_{4}$ and one integration over $\phi_{2}$. This procedure is explained in all detail in the following section where the kinematics is studied. However, as a consequence of these choices, the momentum of the particle 4 could differ with respect to those ones selected for the discrete and finite computational grid. For this reason Eq. A.20 is no longer valid and must be modified. This is done "distributing" the particle 4 over three grid points such that number of particles, energy and momentum are conserved [28]. Once the correct cells are specified, we label them with letters $a, b, c$ and consequently the indexes $i_{a}, k_{a}, i_{b}, k_{b}, i_{c}, k_{c}$ are used to identify the corresponding momentum components on the momentum grid. Hence, Eq. A.23 must be replaced with three equations

$$
\eta_{4 i_{r} k_{r}}^{*}=\int_{V_{i_{r} k_{r}}} d^{3} \mathbf{p} \epsilon_{\mathbf{p}} \eta_{4}(\mathbf{p}), \quad r=\{a, b, c\} .
$$

The emission coefficients in the previous set must be multiplied by the relative weights $x_{a}, x_{b}, x_{c}$. In fact the time derivative of the total energy, number of particles and momentum of the system must be null identically

$$
\begin{gathered}
\dot{\rho}=\sum_{\nu i k} \dot{F}_{\nu i k}=0, \\
\dot{n}=\sum_{\nu i k} \frac{\dot{F}_{\nu i k}}{\epsilon_{\nu i k}}=0, \\
\left\langle\dot{p}_{\|}\right\rangle=\sum_{\nu i k} \frac{\dot{F}_{\nu i k}}{\epsilon_{\nu i k}} p_{\| i}=0,
\end{gathered}
$$

where the notation $\dot{Q}$ means the time derivative $d Q / d t$ due to interactions only. In the previous set of equations, we used only the parallel momentum since the conservation of the orthogonal one is their direct consequence.
The relative weights can be determined uniquely solving the previous system of algebraic equations. Using Dirac Deltas inside integrals, we can rewrite the absorption and emission coefficients as discrete sums

$$
\begin{aligned}
& \chi_{1 i_{1} k_{1}} F_{1 i_{1} k_{1}}=\epsilon_{1 i_{1} k_{1}} \sum_{i_{2} k_{2}} \sum_{i_{3} k_{3}} R_{i_{1} k_{1} i_{2} k_{2} i_{3} k_{3}} F_{2 i_{2} k_{2}} F_{1 i_{1} k_{1}}, \\
& \chi_{2 i_{2} k_{2}} F_{2 i_{2} k_{2}}=\epsilon_{2 i_{2} k_{2}} \sum_{i_{1} k_{1}} \sum_{i_{3} k_{3}} R_{i_{1} k_{1} i_{2} k_{2} i_{3} k_{3}} F_{1 i_{1} k_{1}} F_{2 i_{2} k_{2}}, \\
& \eta_{3 i_{3} k_{3}}^{*}=\epsilon_{3 i_{3} k_{3}} \sum_{i_{1} k_{1}} \sum_{i_{2} k_{2}} R_{i_{1} k_{1} i_{2} k_{2} i_{3} k_{3}} F_{1 i_{1} k_{1}} F_{2 i_{2} k_{2}}, \\
& \eta_{4 i_{r} k_{r}}^{*}=x_{r} \epsilon_{4 i_{r} k_{r}} \sum_{i_{1} k_{1}} \sum_{i_{2} k_{2}} R_{i_{1} k_{1} i_{2} k_{2} i_{3} k_{3}} F_{1 i_{1} k_{1}} F_{2 i_{2} k_{2}},
\end{aligned}
$$

where the index $r$ spans the set $\{a, b, c\}$ and the following coefficient is used

$$
\begin{aligned}
& R_{i_{1} k_{1} i_{2} k_{2} i_{3} k_{3}}=\frac{1}{4(2 \pi)^{3}} \frac{p_{\perp k_{3}}}{\epsilon_{1 i_{1} k_{1}}^{2} \epsilon_{2 i_{2} k_{2}}^{2} \epsilon_{3 i_{3} k_{3}} \epsilon_{4}} \times \\
& \times \sum_{l_{3}} J\left|M_{f i}\right|^{2}\left(\phi_{l_{3}+1 / 2}-\phi_{l_{3}-1 / 2}\right) .
\end{aligned}
$$

The Jacobian, due to the energy to angle change of variable, is given by Eq. A.52 operating the substitution $\phi_{1}=0$

$$
J=\frac{\epsilon_{4} / p_{\perp k_{2}}}{\sin \phi_{2}\left(p_{\perp k_{3}} \cos \phi_{l_{3}}-p_{\perp k_{1}}\right)-p_{\perp k_{3}} \cos \phi_{2} \sin \phi_{l_{3}}} .
$$

Let us note that in Eqs. A.33 and A.34 we used $\epsilon_{4}$ and $\phi_{2}$ because they are obtained from conservation laws and could not be associated with grid values and consequently labeled by indexes. Inserting Eqs. A.29-A.32) into Eqs. (A.26) - A.28) we get a system of three algebraic equations

$$
\begin{aligned}
\sum_{r} x_{r} \epsilon_{4 i_{r} k_{r}} & =\epsilon_{4}, \\
\sum_{r} x_{r} & =1, \\
\sum_{r} x_{r} p_{\| i_{r}} & =p_{\| 4} .
\end{aligned}
$$

Eqs. A.35, A.36 and A.37 state that the global conservation laws are a consequence of the conservation laws for each specific interaction. Now we can solve the system for the 3 unknown $x_{r}$ and its solution reads

$$
\begin{aligned}
& x_{a}=\left[p_{\| 4}\left(\epsilon_{b}-\epsilon_{c}\right)+p_{\| b}\left(\epsilon_{c}-\epsilon_{4}\right)+p_{\| c}\left(\epsilon_{4}-\epsilon_{b}\right)\right] D^{-1}, \\
& x_{b}=\left[p_{\| 4}\left(\epsilon_{c}-\epsilon_{a}\right)+p_{\| a}\left(\epsilon_{4}-\epsilon_{c}\right)+p_{\| c}\left(\epsilon_{a}-\epsilon_{4}\right)\right] D^{-1}, \\
& x_{c}=\left[p_{\| 4}\left(\epsilon_{a}-\epsilon_{b}\right)+p_{\| a}\left(\epsilon_{b}-\epsilon_{4}\right)+p_{\| b}\left(\epsilon_{4}-\epsilon_{a}\right)\right] D^{-1},
\end{aligned}
$$

where the common denominator is

$$
D=p_{\| a}\left(\epsilon_{b}-\epsilon_{c}\right)+p_{\| b}\left(\epsilon_{c}-\epsilon_{a}\right)+p_{\| c}\left(\epsilon_{a}-\epsilon_{b}\right) .
$$


Since $R_{i_{1} k_{1} i_{2} k_{2} i_{3} k_{3}}$ does not depend on the DFs, it can be computed only once before the numerical computation is performed. Looking at Eqs. A.29 -A.32, we easily see that some summations can be also computed as soon as the $R_{i_{1} k_{1} i_{2} k_{2} i_{3} k_{3}}$ are known. Therefore we introduce the following quantities

$$
\begin{aligned}
A_{1 i_{1} k_{1} i_{2} k_{2}} & =\epsilon_{1 i_{1} k_{1}} \sum_{i_{3} k_{3}} R_{i_{1} k_{1} i_{2} k_{2} i_{3} k_{3}}, \\
A_{2 i_{2} k_{2} i_{1} k_{1}} & =\epsilon_{2 i_{2} k_{2}} \sum_{i_{3} k_{3}} R_{i_{1} k_{1} i_{2} k_{2} i_{3} k_{3}}, \\
B_{3 i_{3} k_{3} i_{1} k_{1} i_{2} k_{2}} & =\epsilon_{3 i_{3} k_{3}} R_{i_{1} k_{1} i_{2} k_{2} i_{3} k_{3}}, \\
B_{4 i_{3} k_{3} i_{1} k_{1} i_{2} k_{2}}^{r} & =x_{r} \epsilon_{4 i_{r} k_{r}} R_{i_{1} k_{1} i_{2} k_{2} i_{3} k_{3}},
\end{aligned}
$$

and the final expression for their time derivatives are

$$
\begin{aligned}
\chi_{1 i_{1} k_{1}} F_{1 i_{1} k_{1}} & =\sum_{i_{2} k_{2}} A_{1 i_{1} k_{1} i_{2} k_{2}} F_{i_{1} k_{1}} F_{i_{2} k_{2}}, \\
\chi_{2 i_{2} k_{2}} F_{2 i_{2} k_{2}} & =\sum_{i_{1} k_{1}} A_{2 i_{2} k_{2} i_{1} k_{1}} F_{i_{1} k_{1}} F_{i_{2} k_{2}}, \\
\eta_{3 i_{3} k_{3}}^{*} & =\sum_{i_{1} k_{1}} \sum_{i_{2} k_{2}} B_{3 i_{3} k_{3} i_{1} k_{1} i_{2} k_{2}} F_{i_{1} k_{1}} F_{i_{2} k_{2}}, \\
\eta_{4 i_{r} k_{r}}^{*} & =\sum_{i_{1} k_{1}} \sum_{i_{2} k_{2}} B_{4 i_{3} k_{3} i_{1} k_{1} i_{2} k_{2}}^{r} F_{i_{1} k_{1}} F_{i_{2} k_{2}} .
\end{aligned}
$$

The emission coefficients requires $(I \times K)^{2}$ operations for each time step which must be multiplied by another factor $(I \times K)$ due to the computation of the analytical jacobian needed by the adopted method. As a result we have a total of about $(I \times K)^{3}$ calculations at each time step which puts strong limits on the maximum number of grid points $I$ and $K$.

In order to describe processes with different timescales we use the Gear's method for stiff ODE's used in [28]. In fact, this numerical approach has an adaptive time step which becomes small when the DF time derivative is large, on the contrary it becomes large when the DF time derivative is small.

\section{Appendix A.3. Two particle kinematics}

The interaction between two particles, 1 and 2, that gives the particle 3 and 4 as a product can be represented schematically as follows

$$
1+2 \rightarrow 3+4
$$

Each particle has 3 phase space coordinates $\left(p_{\nu_{\|}}, p_{\nu_{\perp}}, \phi_{\nu}\right)$, therefore we have a total of 12 variables for the 2-particles interactions we are dealing with. Since each interaction conserves momentum and energy, they reduce to 8 independent degrees of freedom. That means that 4 quantities can be determined uniquely once the others are specified. For us, the 8 independent variables are $p_{1_{\|}}, p_{1_{\perp}}, \phi_{1}, p_{2_{\|}}$, $p_{2_{\perp}}, p_{3_{\|}}, p_{3_{\perp}}, \phi_{3}$; then $p_{4_{\|}}, p_{4_{\perp}}, \phi_{4}, \phi_{2}$ are functions of the previous ones.
The conservation of the parallel momentum gives us the corresponding component of the 4-th particle

$$
p_{4_{\|}}=p_{1_{\|}}+p_{2_{\|}}-p_{3_{\|}} .
$$

The orthogonal momentum for the same particle can be worked out using the energy conservation law

$$
\epsilon_{1}+\epsilon_{2}=\epsilon_{3}+\epsilon_{4}
$$

and using the definition of the energy given by Eq. (11) as follows

$$
p_{4_{\perp}}=\sqrt{\left(\epsilon_{1}+\epsilon_{2}-\epsilon_{3}\right)^{2}-p_{4_{\|}}^{2}-m_{4}^{2}}
$$

where $p_{4_{\|}}$has been obtained in Eq. (A.40). From the conservation of the orthogonal momentum we have the following relations

$$
\begin{aligned}
p_{1_{\perp}} \cos \phi_{1}+p_{2_{\perp}} \cos \phi_{2} & =p_{3_{\perp}} \cos \phi_{3}+p_{4_{\perp}} \cos \phi_{4} \\
p_{1_{\perp}} \sin \phi_{1}+p_{2_{\perp}} \sin \phi_{2} & =p_{3_{\perp}} \sin \phi_{3}+p_{4_{\perp}} \sin \phi_{4}
\end{aligned}
$$

from which we can write down analytical expressions for $\phi_{4}$ and $\phi_{2}$. Unfortunately for the previous system of equations we have two valid solutions. For $\phi_{2}$ we have the following equation

$$
a \cos \phi_{2}+b \sin \phi_{2}+c=0
$$

where the coefficients are given by

$$
\begin{aligned}
a & =p_{1_{\perp}} \cos \phi_{1}-p_{3_{\perp}} \cos \phi_{3}, \\
b & =p_{1_{\perp}} \sin \phi_{1}-p_{3_{\perp}} \sin \phi_{3}, \\
c & =\frac{a^{2}+b^{2}+p_{2_{\perp}}^{2}-p_{4_{\perp}}^{2}}{2 p_{2_{\perp}}} .
\end{aligned}
$$

The solution of eq. A.45 are given by the following conditions

- if $b \neq 0, a^{2}+b^{2} \neq 0, c=a$

$$
\Rightarrow \phi_{2}=-2 \arctan \left(\frac{a}{b}\right) \text {, }
$$

- if $b=0, c=a \quad \Rightarrow \quad \phi_{2}=\pi$,

- if $a \neq c, a^{2}+b^{2}-a c-b \sqrt{a^{2}+b^{2}-c^{2}} \neq 0$

$$
\Rightarrow \quad \phi_{2}=2 \arctan \left(\frac{b-\sqrt{a^{2}+b^{2}-c^{2}}}{a-c}\right) \text {, }
$$

- if $a \neq c, a^{2}+b^{2}-a c+b \sqrt{a^{2}+b^{2}-c^{2}}$

$$
\Rightarrow \quad \phi_{2}=2 \arctan \left(\frac{b+\sqrt{a^{2}+b^{2}-c^{2}}}{a-c}\right) .
$$

Once $\phi_{2}$ has been chosen, $\phi_{4}$ can be easily obtained from the Eqs. A.43 and (A.44).

The Jacobian of Eq. A.34 has been computed using the following identity for the Dirac Delta

$$
\delta(f(x))=\sum_{i} \frac{\delta\left(x-x_{i}\right)}{\left|(d f / d x)_{x_{i}}\right|},
$$


where $f$ is a function such that $f\left(x_{i}\right)=0$. In our framework the function inside the Dirac delta is given by the energy conservation

$$
f\left(\phi_{2}\right)=\epsilon_{1}+\epsilon_{2}-\epsilon_{3}-\epsilon_{4}\left(\phi_{2}\right),
$$

where $\phi_{2}$ is now the independent variable. From the previous equation we compute its derivative with respect to $\phi_{2}$ and the value $\phi_{2}^{*}$ such that $f\left(\phi_{2}^{*}\right)=0$. Rewriting explicitly Eq. (A.49) we have that

$$
\delta\left(f\left(\phi_{2}\right)\right)=\frac{\delta\left(\phi_{2}-\phi_{2}^{*}\right)}{\left|\left(d f / d \phi_{2}\right)_{\phi_{2}^{*}}\right|}=J \delta\left(\phi_{2}-\phi_{2}^{*}\right),
$$

where the explicit equation for $J$ is

$$
J=\frac{\epsilon_{4}}{p_{2_{\perp}}\left|\sin \left(\phi_{2}-\phi_{4}\right)\right|}
$$

\section{Acknowledgments}

AB is supported by the Erasmus Mundus Joint Doctorate Program by Grant Number 2010-1816 from the EACEA of the European Commission.

\section{References}

[1] F. Sauter, Über das Verhalten eines Elektrons im homogenen elektrischen Feld nach der relativistischen Theorie Diracs., Zeits. Phys. 69 (1931) 742-+.

[2] W. Heisenberg, H. Euler, Zeits. Phys. 98 (1935) 714-+.

[3] J. Schwinger, On Gauge Invariance and Vacuum Polarization, Phys. Rev. 82 (1951) 664-679.

[4] C. Bula, K. T. McDonald, E. J. Prebys, C. Bamber, S. Boege, T. Kotseroglou, A. C. Melissinos, D. D. Meyerhofer, W. Ragg, D. L. Burke, R. C. Field, G. Horton-Smith, A. C. Odian, J. E. Spencer, D. Walz, S. C. Berridge, W. M. Bugg, K. Shmakov, A. W. Weidemann, Observation of Nonlinear Effects in Compton Scattering, Physical Review Letters 76 (1996) 3116-3119.

[5] D. L. Burke, R. C. Field, G. Horton-Smith, J. E. Spencer, D. Walz, S. C. Berridge, W. M. Bugg, K. Shmakov, A. W. Weidemann, C. Bula, K. T. McDonald, E. J. Prebys, C. Bamber, S. J. Boege, T. Koffas, T. Kotseroglou, A. C. Melissinos, D. D. Meyerhofer, D. A. Reis, W. Ragg, Positron Production in Multiphoton Light-by-Light Scattering, Physical Review Letters 79 (1997) 1626-1629.

[6] A. Di Piazza, C. Müller, K. Z. Hatsagortsyan, C. H. Keitel, Extremely high-intensity laser interactions with fundamental quantum systems, Reviews of Modern Physics 84 (2012) 11771228. arXiv:1111.3886, doi:10.1103/RevModPhys.84.1177

[7] A. R. Bell, J. G. Kirk, Possibility of Prolific Pair Production with High-Power Lasers, Physical Review

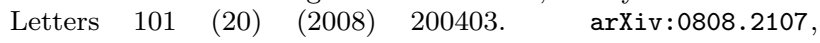
doi:10.1103/PhysRevLett.101.200403

[8] S. S. Bulanov, T. Z. Esirkepov, A. G. R. Thomas, J. K. Koga, S. V. Bulanov, Schwinger Limit Attainability with Extreme Power Lasers, Physical Review Letters 105 (22) (2010) 220407. arXiv:1007.4306, doi:10.1103/PhysRevLett.105.220407.

[9] A. M. Fedotov, N. B. Narozhny, G. Mourou, G. Korn, Limitations on the Attainable Intensity of High Power Lasers, Physical Review Letters 105 (8) (2010) 080402. arXiv:1004.5398, doi:10.1103/PhysRevLett.105.080402

[10] V. V. Usov, Bare Quark Matter Surfaces of Strange Stars and $e^{+} e^{-}$Emission, Phys. Rev. Lett. 80 (1998) 230-233. arXiv:arXiv:astro-ph/9712304
[11] C. Alcock, E. Farhi, A. Olinto, Strange stars, ApJ 310 (1986) 261-272. doi:10.1086/164679.

[12] R. Belvedere, D. Pugliese, J. A. Rueda, R. Ruffini, S.-S. Xue, Neutron star equilibrium configurations within a fully relativistic theory with strong, weak, electromagnetic, and gravitational interactions, Nuclear Physics A 883 (2012) 1-24. arXiv:1202.6500 doi:10.1016/j.nuclphysa.2012.02.018

[13] R. Ruffini, G. Vereshchagin, S.-S. Xue, Electron-positron pairs in physics and astrophysics, Physics Reports 487 (2010) 1-140.

[14] V. V. Usov, Thermal Emission from Bare Quark Matter Surfaces of Hot Strange Stars, ApJ 550 (2001) L179-L182. arXiv:arXiv:astro-ph/0103361, doi:10.1086/319639

[15] Y. Kluger, J. M. Eisenberg, B. Svetitsky, F. Cooper, E. Mottola, Pair production in a strong electric field, Phys. Rev. Lett. 67 (1991) 2427-2430. doi:10.1103/PhysRevLett.67.2427

[16] Y. Kluger, J. M. Eisenberg, B. Svetitsky, F. Cooper, E. Mottola, Fermion pair production in a strong electric field, Phys. Rev. D45 (1992) 4659-4671. doi:10.1103/PhysRevD.45.4659.

[17] T. Matsui, K. Kajantie, Decay of strong color electric field and thermalization in ultra-relativistic nucleus-nucleus collisions, Physics Letter B 164 (1985) 373.

[18] S. A. Smolyansky, G. Roepke, S. Schmidt, D. Blaschke, V. D. Toneev, A. V. Prozorkevich, Dynamical derivation of a quantum kinetic equation for particle production in the Schwinger mechanism, ArXiv High Energy Physics - Phenomenology eprintsarXiv:arXiv: hep-ph/9712377.

[19] S. Schmidt, D. Blaschke, G. Röpke, S. A. Smolyansky, A. V. Prozorkevich, V. D. Toneev, A Quantum Kinetic Equation for Particle Production in the Schwinger Mechanism, International Journal of Modern Physics E 7 (1998) 709-722. arXiv:arXiv:hep-ph/9809227 doi:10.1142/S0218301398000403

[20] G. Gatoff, A. K. Kerman, T. Matsui, Flux-tube model for ultrarelativistic heavy-ion collisions: Electrohydrodynamics of a quark-gluon plasma, Phys. Rev. D36 (1987) 114-129. doi:10.1103/PhysRevD.36.114

[21] Y. Kluger, E. Mottola, J. M. Eisenberg, Quantum Vlasov equation and its Markov limit, Phys. Rev. D58 (12) (1998) 125015. arXiv:arXiv:hep-ph/9803372 doi:10.1103/PhysRevD.58.125015

[22] J. C. R. Bloch, V. A. Mizerny, A. V. Prozorkevich, C. D. Roberts, S. M. Schmidt, S. A. Smolyansky, D. V. Vinnik, Pair creation: Back reactions and damping, Phys. Rev. D60 (11) (1999) 116011. arXiv:arXiv:nucl-th/9907027 doi:10.1103/PhysRevD.60.116011

[23] D. V. Vinnik, A. V. Prozorkevich, S. A. Smolyansky, V. D. Toneev, M. B. Hecht, C. D. Roberts, S. M. Schmidt, Plasma production and thermalisation in a strong field, European Physical Journal C 22 (2001) 341-349. arXiv:arXiv:nucl-th/0103073, doi:10.1007/s100520100787

[24] D. B. Blaschke, V. V. Dmitriev, G. Röpke, S. A. Smolyansky, BBGKY kinetic approach for an $\mathrm{e}^{-} \mathrm{e}^{+} \gamma$ plasma created from the vacuum in a strong laser-generated electric field: The onephoton annihilation channel, Phys. Rev. D84 (8) (2011) 085028. arXiv:1105.5397 doi:10.1103/PhysRevD.84.085028

[25] R. Ruffini, L. Vitagliano, S.-S. Xue, On plasma oscillations in strong electric fields, Phys. Lett. B 559 (2003) 12-19. arXiv:arXiv:astro-ph/0302549

[26] R. Ruffini, G. V. Vereshchagin, S.-S. Xue, Vacuum polarization and plasma oscillations, Phys. Lett. A 371 (2007) 399-405.

[27] A. Benedetti, W.-B. Han, R. Ruffini, G. V. Vereshchagin, On the frequency of oscillations in the pair plasma generated by a strong electric field, Physics Letters B 698 (2011) 75-79. arXiv:1102.4287 doi:10.1016/j.physletb.2011.02.050

[28] A. G. Aksenov, R. Ruffini, G. V. Vereshchagin, Thermalization of the mildly relativistic plasma, Phys. Rev. D79 (4) (2009) 043008. arXiv:0901.4837 doi:10.1103/PhysRevD.79.043008

[29] A. G. Aksenov, M. Milgrom, V. V. Usov, Structure of Pair Winds from Compact Objects with Application to Emission from Hot Bare Strange Stars, ApJ 609 (2004) 363-377. 
arXiv:arXiv:astro-ph/0309014, doi:10.1086/421006

[30] S. R. de Groot, W. A. van Leeuwen, C. G. van Weertn, Relativistic kinetic theory. Principles and Applications, NorthHolland, Amsterdam, 1980.

[31] A. G. Aksenov, R. Ruffini, G. V. Vereshchagin, Thermalization of Nonequilibrium Electron-Positron-Photon Plasmas, Phys. Rev. Lett. 99 (12) (2007) 125003-+.

[32] D. Mihalas, B. W. Mihalas, Foundations of Radiation Hydrodynamics, New York, Oxford University Press, 1984.

[33] L. D. Landau, E. M. Lifshitz, Physical Kinetics, Elsevier, 1981. 